\title{
LAMP3 promotes the invasion of osteosarcoma cells via SPP1 signaling
}

\author{
YU LI ${ }^{1}$, WEI DU ${ }^{2}$, JIAN HAN ${ }^{3}$ and JUNBO GE ${ }^{1}$ \\ Departments of ${ }^{1}$ Bone Trauma, ${ }^{2}$ Spine Branch and ${ }^{3}$ Bone Oncology, Yantaishan Hospital, Yantai, Shandong 264000, P.R. China
}

Received October 25, 2016; Accepted June 9, 2017

DOI: $10.3892 / \mathrm{mmr} .2017 .7349$

\begin{abstract}
Osteosarcoma is the most common type of primary bone cancer in children and young adults. The prognosis of osteosarcoma is very poor when it is diagnosed with metastasis. Lysosomal-associated membrane protein 3 (LAMP3) is a tumor-specific protein induced by hypoxia, which stimulates invasion and metastasis of various cancer cells via hypoxia-inducible factor (HIF). A previous study from our group has reported that expression of LAMP3 is significantly increased in lung metastatic osteosarcoma compared with primary osteosarcoma using microarray analysis, suggesting that LAMP3 may be involved in metastatic osteosarcoma. The present study therefore aimed to investigate the role of LAMP3 in osteosarcoma metastasis. Knockdown of LAMP3 decreased the invasion of two osteosarcoma cell lines in vitro. Furthermore, knockdown of LAMP3 increased the expression of secreted phosphoprotein 1 (SPP1), cadherin 1, and keratin 19, while it decreased the expression of matrix metallopeptidase 2, collagen type III $\alpha 1$, twist family bHLH transcription factor 1 and cadherin 2. Concurrent knockdown of SPP1 and LAMP3 attenuated the changes in gene expression profile induced by LAMP3 knockdown alone. Gene ontology and KEGG analysis demonstrated that SPP1 was involved in cell adhesion, focal adhesion, and extracellular matrix-receptor interaction. In conclusion, the present results suggest that LAMP3 may be involved in the invasion and metastasis of osteosarcoma via regulating signaling downstream of SPP1. Thus, LAMP3/SPP1 signaling may serve as a potential target in the future to prevent osteosarcoma metastasis.
\end{abstract}

\section{Introduction}

Osteosarcoma is the most common type of primary bone cancer. Most osteosarcomas are detected at the end of long

Correspondence to: Mr. Junbo Ge, Department of Bone Trauma, Yantaishan Hospital, 91 Jiefang Road, Yantai, Shandong 264000, P.R. China

E-mail: gejunbo_sd@163.com

Key words: osteosarcoma, lysosomal-associated membrane protein 3 , secreted phosphoprotein 1 , invasion bone in children and adolescence when they are most rapidly growing (1). Despite the advances in chemotherapy and radiation treatments, the survival rate has remained low in the past two decades because of the fatal lung metastasis at the late stage of this disease (2). The mechanisms underlying the metastasis remain largely unknown. Understanding the mechanisms will help develop strategies for preventing metastasis of osteosarcoma and improving the survival rate of patients.

Lysosomal-associated membrane protein 3 (LAMP3) is a tumor-specific protein, which was originally isolated from lung tissue (3). Several lines of evidence indicate that LAMP3 is upregulated in different types of cancer, including breast cancer, uterine cervical cancer, esophageal and laryngeal squamous cell carcinoma, and gastrointestinal cancer (4-8). High expression of LAMP3 is associated with poor prognosis and low survival rate in patients with esophageal and laryngeal squamous cell carcinomas $(7,8)$. LAMP3 has also been demonstrated to promote metastasis of uterine cervical cancer and to have important roles in chemotherapy resistance of breast cancer $(4,9)$. In a previously published study from our group, LAMP3 was demonstrated to be highly expressed in lung metastasis of osteosarcoma compared with primary osteosarcoma tissues (10). However, the exact role of LAMP3 and its mechanism in osteosarcoma invasion and metastasis remain unclear.

The present study demonstrated that knockdown of LAMP3 decreased osteosarcoma cell invasion via regulating multiple genes involved in cancer cell invasion. Concurrent knockdown of LAMP3 and secreted phosphoprotein 1 (SPP1), the most upregulated gene induced by LAMP3 knockdown, attenuated the changes of gene expression mediated by knockdown of LAMP3. SPP1 was predicted to be involved in functions and signaling pathways related to cancer cell invasion. Taken together, these results demonstrated that LAMP3 may be important in invasion of osteosarcoma via regulating the SPP1 signaling pathway, suggesting that LAMP3 and SPP1 may serve as potential targets to prevent osteosarcoma metastasis and improve the survival rate of patients.

\section{Materials and methods}

Cell culture and transfection. OS-732 and U2OS cell lines were purchased from the American Type Culture Collection (Manassas, VA, USA) and maintained in Dulbecco's modified Eagle's medium (Invitrogen; Thermo Fisher Scientific, Inc., 
Waltham, MA, USA) supplemented with $10 \%$ fetal bovine serum (Invitrogen; Thermo Fisher Scientific, Inc.), $2 \mathrm{mM}$ glutamine, $100 \mathrm{U} / \mathrm{ml}$ penicillin and $100 \mu \mathrm{g} / \mathrm{ml}$ streptomycin. Cells were maintained at $37^{\circ} \mathrm{C}$ in a humidified atmosphere containing $5 \% \mathrm{CO}_{2}$. For the knockdown of LAMP3 and SPP1, 20 nM siLAMP3 (L-004716-01-0005), siSPP1 (L-012558-00-0005) and siControl (D-001810-01-05; all from GE Healthcare Dharmacon, Inc., Lafayette, CO, USA) were transfected to cells using HiPerFect transfection reagent (Qiagen GmbH, Hilden, Germany), according to manufacturer's instructions. Then, 2 days after transfection of siRNAs, the medium was replaced with fresh complete medium and the cells were ready for the experiments.

Reverse transcription-quantitative polymerase chain reaction (RT-qPCR). Total RNA was extracted from cells using TRIzol reagent (Invitrogen; Thermo Fisher Scientific, Inc.). Total RNA $(1 \mu \mathrm{g})$ was treated with DNase I (Invitrogen; Thermo Fisher Scientific, Inc.) and cDNA was synthesized using the SuperScript III First-Strand Synthesis kit (Invitrogen; Thermo Fisher Scientific, Inc.). qPCR was performed in a 7900 HT real time PCR system (Applied Biosystems; Thermo Fisher Scientific, Inc.) using SYBR Green Real-Time PCR Master Mix (Sigma-Aldrich; Merck KGaA, Darmstadt, Germany). PCR conditions were: Initial denaturation, $95^{\circ} \mathrm{C}, 30 \mathrm{sec}$; 40 cycles: (Step 1) $95^{\circ} \mathrm{C}, 5 \mathrm{sec}$; (Step 2) $58^{\circ} \mathrm{C}, 15 \mathrm{sec}$; (Step 3) $72^{\circ} \mathrm{C}, 10 \mathrm{sec}$. GAPDH was used as an internal control. Relative mRNA levels were calculated using the $2^{-\Delta \Delta \mathrm{Cq}}$ method (10). The sequences of primers are given in Table I.

Transwell invasion assay. Cell invasion assay was carried out using a 24-well Transwell culture system. The upper side of the filter was coated with $100 \mu 10.25 \mathrm{mg} / \mathrm{ml}$ Matrigel at $4^{\circ} \mathrm{C}$ for overnight. The lower chamber was filled with complete culture medium (10\% FBS). A total of $5 \times 10^{4}$ cells were plated into the upper part of the Transwell chamber (FBS free) and incubated for $24 \mathrm{~h}$. Following incubation, the non-invaded cells on the upper side of the membrane were removed by cotton swabs. The invaded cells on the lower side of the insert filter were fixed with $10 \%$ buffered formaldehyde and stained with $1 \%$ crystal violet solution. The invasive ability was determined by counting the cells that invaded to the lower side of the membrane under a light microscope (Olympus Corporation, Tokyo, Japan). A total of three random fields were chosen to manually count the cell numbers; two replicates were performed per experiment and three independent experiments were performed.

Western blotting. The protein expression levels of LAMP3, E-cadherin, and $\beta$-actin were analyzed by western blot assay. Following treatments, cells were harvested in radioimmunoprecipitation assay lysis buffer (Beyotime Institute of Biotechnology, Shanghai, China) supplemented with protease inhibitor cocktail (Sigma-Aldrich; Merck KGaA). Equal amount of protein $(\sim 20 \mu \mathrm{g})$ was resolved in $12 \%$ SDS-PAGE and transferred onto polyvinylidene fluoride membranes. Following blocking with 5\% non-fat dry milk in TBS/0.1\% Tween-20 (TBST) buffer, membranes were incubated with primary antibodies at $4^{\circ} \mathrm{C}$ overnight. Following three washes with TBST, the membranes were incubated with horseradish peroxidase (HRP)-conjugated secondary antibodies for $1 \mathrm{~h}$ at room temperature. Following washing, the membranes were developed using chemiluminescence substrate (RPN2232; GE Healthcare Life Sciences, Little Chalfont, UK) and imaged. The antibodies used were: Mouse anti- $\beta$-actin (ab8227; 1:5,000; Abcam), anti-mouse HRP-conjugated IgG (A9044; 1:5,000; Sigma-Aldrich; Merck KGaA), anti-rabbit HRP-conjugated IgG (A0545; 1:5,000; Sigma-Aldrich; Merck KGaA), anti-E-Cadherin (3195; 1:1,000; Cell Signaling Technology, Inc., Danvers, MA, USA), anti-LAMP3 (ab83659; 1:1,000; Abcam).

Gene Ontology (GO) and Kyoto Encyclopedia of Genes and Genomes (KEGG) pathway analysis. The R packages GO.db and KEGGREST (11) were used to detect GO categories and KEGG (http://www.genome.jp/kegg/) pathways that were significantly enriched in the differentially expressed genes, as previously published (12). $\mathrm{P}<0.01$ indicated significantly enriched GO terms, whereas $\mathrm{P}<0.05$ indicated significantly enriched KEGG pathways.

Statistical analysis. GraphPad InStat 3 (GraphPad Software, Inc., La Jolla, CA, USA) was used for statistical analysis. Data are presented as means \pm standard error of the mean of $\geq 3$ independent experiments. Significance of differences between two groups was determined by student's t-test and one-way analysis of variation with post hoc Tukey-Kramer Multiple Comparisons Test was used to calculate the significant differences among $\geq 3$ groups. $\mathrm{P}<0.05$ was considered to indicate a statistically significant difference.

\section{Results}

Knockdown of LAMP3 decreases the invasion of osteosarcoma cells. In view of the upregulation and premetastatic effect of LAMP3 in uterine cervical cancer and breast cancer $(4,6)$, the role of LAMP3 in osteosarcoma metastasis was investigated. In a previously published study from our group, microarray data (accession no. GSE14359) from primary and lung metastatic osteosarcoma samples were downloaded from the Gene Expression Omnibus database (http://www.ncbi.nlm.nih. gov/geo) and analyzed, and the results revealed that LAMP3 was among the top 10 upregulated genes in the lung metastatic tissues (10), suggesting that LAMP3 may be involved in osteosarcoma metastasis.

To investigate the function of LAMP3 on osteosarcoma cell invasion, the critical first step of the metastatic cascade, small interfering RNA (siRNA) silencing of LAMP3 was employed in two osteosarcoma cell lines, OS-732 and U2OS. First, the successful knockdown of LAMP3 was confirmed by western blotting in the two cell lines (Fig. 1A). Then, the invasive ability of osteosarcoma cells transfected with siControl or siLAMP3 was compared using an in vitro Transwell invasion assay. LAMP3 knockdown significantly decreased the number of cells invading the matrigel in both cell lines, compared with cells transfected with siControl (Fig. 1B). It is well-known that loss of E-cadherin is associated with increase of cancer cell invasion. Therefore, the effect of LAMP3 knockdown on E-cadherin expression was also examined by western blotting. The results demonstrated that LAMP3 knockdown by siRNA resulted in a marked increase in E-cadherin protein expression 
Table I. Sequences of primers used.

\begin{tabular}{|c|c|c|}
\hline Gene symbol & Forward primer $\left(5^{\prime}-3^{\prime}\right)$ & Reverse primer $\left(5^{\prime}-3^{\prime}\right)$ \\
\hline MMP2 & TACAGGATCATTGGCTACACACC & GTCACATCGCTCCAGACT \\
\hline TWIST1 & GTCCGCAGTCTTACGAGGAG & GCTTGAGGGTCTGAATCTTGCT \\
\hline CDH1 & ATTTTTCCCTCGACACCCGAT & TCCCAGGCGTAGACCAAGA \\
\hline $\mathrm{CDH} 2$ & TCAGGCGTCTGTAGAGGCTT & ATGCACATCCTTCGATAAGACTG \\
\hline KRT19 & AACGGCGAGCTAGAGGTGA & GGATGGTCGTGTAGTAGTGGC \\
\hline COL3A1 & GGAGCTGGCTACTTCTCGC & GGGAACATCCTCCTTCAACAG \\
\hline CAV2 & AAGACCTGCCTAATGGTTCTGC & CTCGTACACAATGGAGCAATGAT \\
\hline DSP & GCAGGATGTACTATTCTCGGC & CCTGGATGGTGTTCTGGTTCT \\
\hline MST1R & CTTTGACGTGAAGTACGTGGT & CGTATGGCTACAAACACAGCAC \\
\hline OCLN & ACAAGCGGTTTTATCCAGAGTC & GTCATCCACAGGCGAAGTTAAT \\
\hline RGS2 & AAGATTGGAAGACCCGTTTGAG & GCAAGACCATATTTGCTGGCT \\
\hline TFPI2 & CTGGGGCTGTCGATTCTGC & TCTCCGCGTTATTTCCTGTTG \\
\hline AHNAK & TACCCTTCCTAAGGCTGACATT & TTGGACCCTTGAGTTTTGCAT \\
\hline BMP1 & GGGTCATCCCCTTTGTCATTG & GCAAGGTCGATAGGTGAACACA \\
\hline FN1 & CGGTGGCTGTCAGTCAAAG & AAACCTCGGCTTCCTCCATAA \\
\hline FOXC2 & ССТCCTGGTATCTCAACCACA & GAGGGTCGAGTTCTCAATCCC \\
\hline GSC & AACGCGGAGAAGTGGAACAAG & CTGTCCGAGTCCAAATCGC \\
\hline ITGA5 & GGCTTCAACTTAGACGCGGAG & TGGCTGGTATTAGCCTTGGGT \\
\hline MMP9 & TGTACCGCTATGGTTACACTCG & GGCAGGGACAGTTGCTTCT \\
\hline MSN & GAGGATGTGTCCGAGGAATTG & GTCTCAGGCGGGCAGTAAA \\
\hline GAPDH & ACAACTTTGGTATCGTGGAAGG & GCCATCACGCCACAGTTTC \\
\hline
\end{tabular}

A
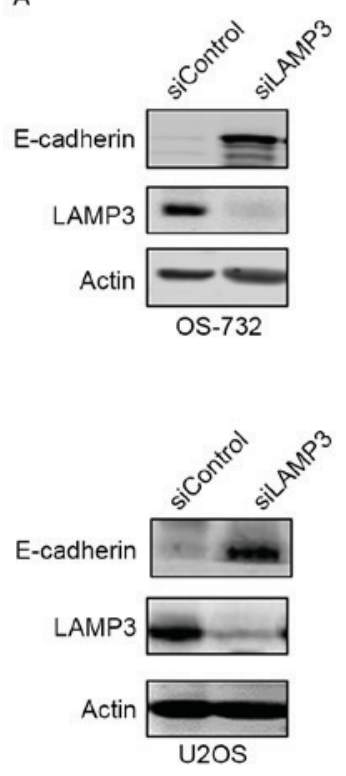

B

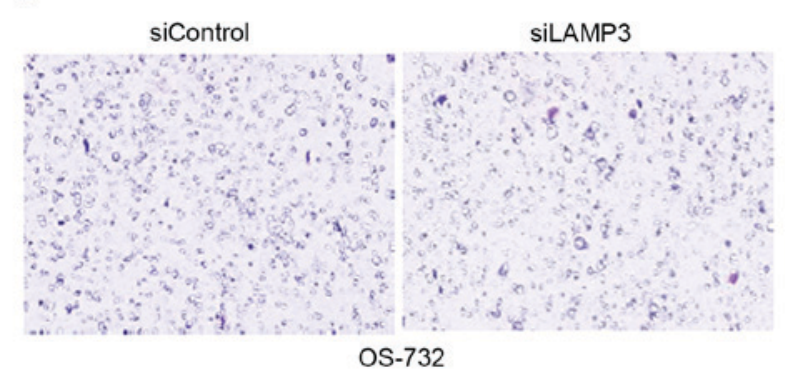

OS-732

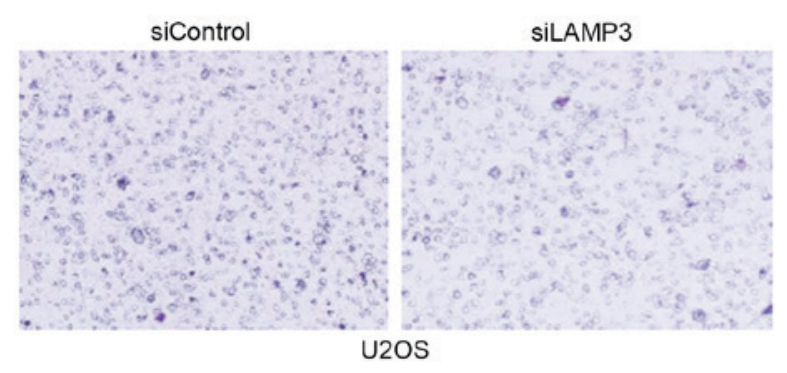

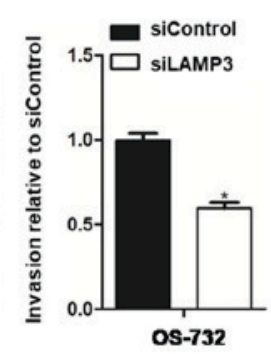

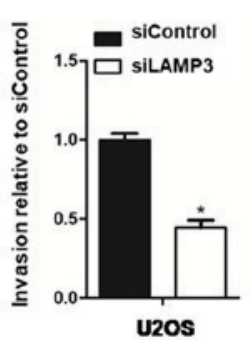

Figure 1. Knockdown of LAMP3 decreases osteosarcoma cell invasion. OS-732 and U2OS osteosarcoma cells were transfected with either negative control siRNA (siControl) or siRNA targeting LAMP3 (siLAMP3). (A) Protein expression levels of LAMP3 and E-cadherin were examined by western blotting in the two cells lines. Actin was used as an internal loading control. (B) Representative images (magnification, x10) and quantification from Transwell invasion assays in the two cell lines. ${ }^{*} \mathrm{P}<0.05$ vs. siControl. LAMP3, lysosomal-associated membrane protein 3; si, small interfering.

levels compared with cells transfected with siControl (Fig. 1A), which is consistent with a decreased invasion ability.

Knockdown of LAMP3 increases the mRNA expression of SPP1. To elucidate the downstream signaling of LAMP3 in the regulation of cell invasion, the EMT-related gene expression profile, summarized by PCR array on the Qiagen website (http://www.sabiosciences.com), was examined using RT-qPCR after transfection of siLAMP3 in osteosarcoma cells. As presented in Fig. 2, SPP1 was the top upregulated 


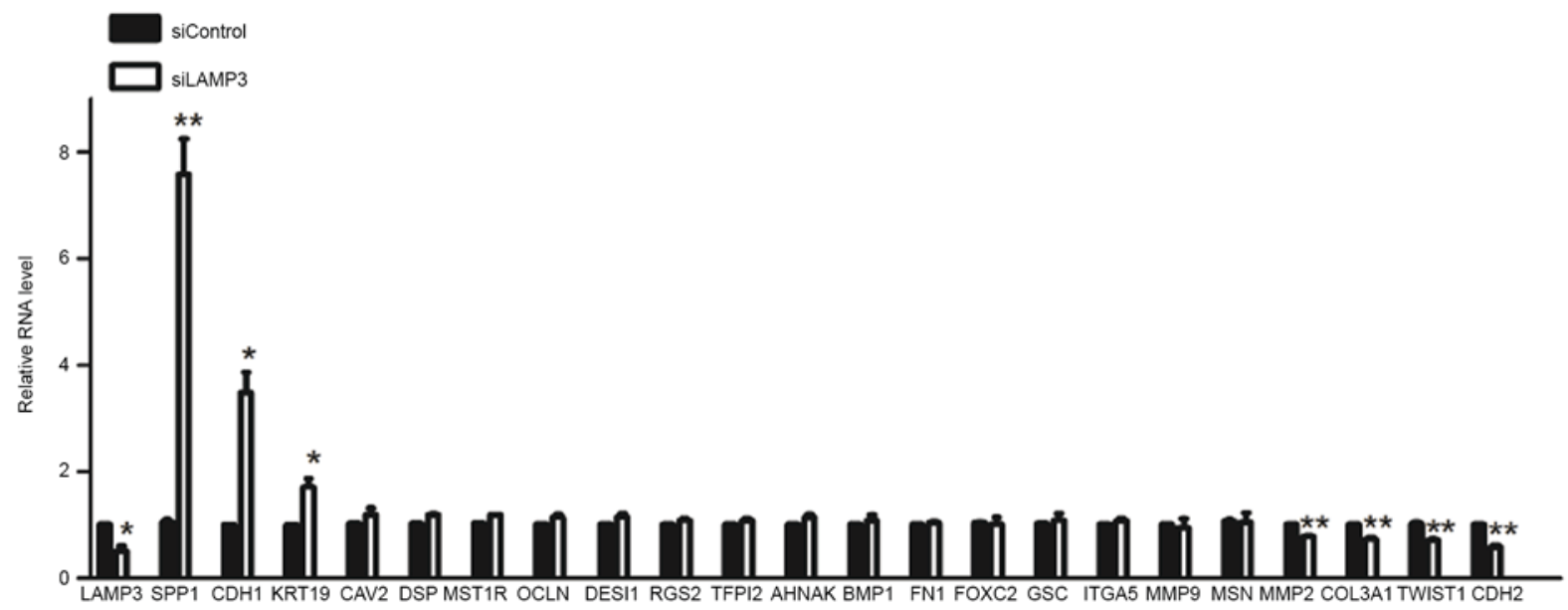

Figure 2. Gene expression profile following LAMP3 knockdown in U2OS. Reverse transcription-quantitative polymerase chain reaction was employed to analyze the expression changes of genes associated with cancer cell invasion and metastasis following knockdown of LAMP3 (siLAMP3). Results are presented relative to the mRNA levels of the cells transfected with control siRNA (siControl). ${ }^{*} \mathrm{P}<0.05$ and $^{* *} \mathrm{P}<0.01$ vs. siControl. LAMP3, lysosomal-associated membrane protein 3 ; si, small interfering.
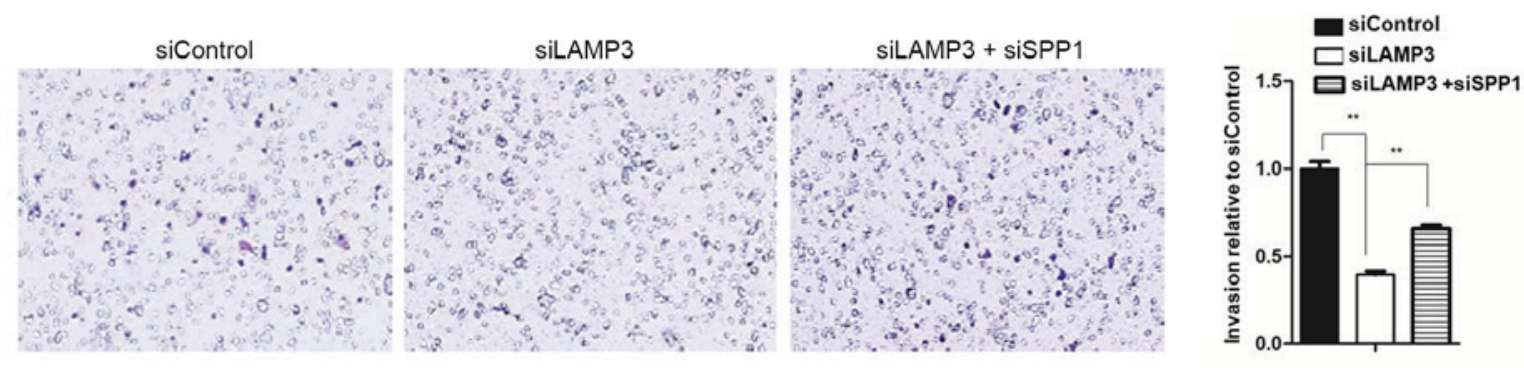

Figure 3. Knockdown of SPP1 partially rescues the inhibition of invasion induced by LAMP3 silencing. U2OS cells were transfected with control siRNA (siControl), LAMP3-specific siRNA (siLAMP3) and/or SPP1-specific siRNA (siSPP1) and their invasive ability was examined by Transwell invasion assay. Representative images (magnification, x10) and quantification are presented. ${ }^{*} \mathrm{P}<0.05$ and ${ }^{* *} \mathrm{P}<0.01$ vs. siControl. SPP1, secreted phosphoprotein 1; LAMP3, lysosomal-associated membrane protein 3 ; si, small interfering.

gene induced by LAMP3 knockdown. Consistent with the upregulation of E-cadherin presented in Fig. 1, an increase in the mRNA expression of the CDH1 gene was also observed following LAMP3 knockdown (Fig. 2). KRT19 was also significantly increased in osteosarcoma cells following LAMP3 knockdown. In addition, expression of genes that are known to be involved in tumor cell invasion, including MMP2, COL3A1, TWIST1, and CDH2, were significantly downregulated in siLAMP3 transfected cells (Fig. 2). The present results suggest that these genes may serve as the downstream signaling mediators of LAMP3-mediated osteosarcoma metastasis.

Inhibition of SPP1 partially rescues the effect of LAMP3 knockdown. To further confirm the role of SPP1 in LAMP3-mediated promotion of osteosarcoma invasion, LAMP3 and SPP1 were silenced concurrently and the invasive ability of osteosarcoma cells was examined. As illustrated in Fig. 3, the number of invaded cells was reduced following LAMP3 knockdown, while co-transfection of SPP1-specific siRNA partially but significantly reversed this effect. Furthermore, the effect of SPP1 knockdown was investigated on the siLAMP3-mediated gene expression changes in osteosarcoma cells. Similar to the in vitro invasion results, concurrent knockdown of SPP1 significantly attenuated the upregulation of CDH1 and KRT19 and the downregulation of MMP2, COL3A1, TWIST1 and $\mathrm{CDH} 2$ induced by LAMP3 knockdown, compared with siLAMP3-trasfection alone (Fig. 4).

SPP1 is associated with cell invasion pathways. Finally, GO and KEGG enrichment pathway analysis of SPP1 in a public online human osteosarcoma patient database (http://osteosarcoma-db.uni-muenster.de/genesearch.php?gene2search=6696) were employed to investigate the cellular functions and signaling pathways in which SPP1 may be involved. The data presented in Fig. 5 demonstrated that SPP1 was involved in $21 \mathrm{GO}$ terms and $3 \mathrm{KEGG}$ pathways. The top significantly enriched GO terms included osteoblast differentiation, cell adhesion, and inflammatory response. The top KEGG pathways involving SPP1 were focal adhesion, extracellular matrix (ECM)-receptor interaction and Toll-like receptor signaling pathway. These results suggest a functional role of SPP1 in the regulation of cell adhesion, loss of which is critical for the initiation of the metastatic cascade.

\section{Discussion}

Osteosarcoma is the most common type of primary bone cancer. Despite of aggressive surgery and intensive 

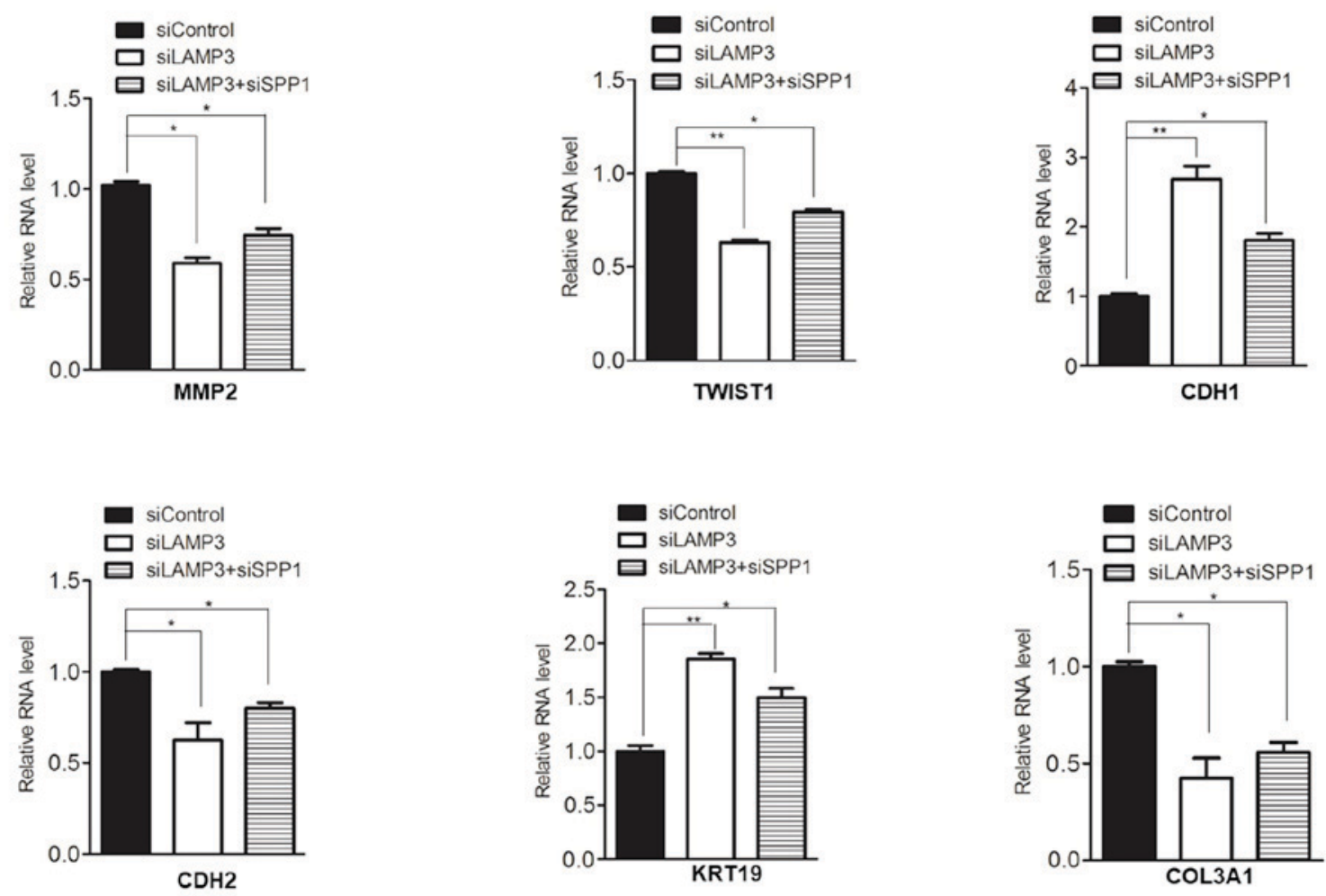

Figure 4. Knockdown of SPP1 partially rescues the gene expression changes induced by LAMP3 silencing. U2OS cells were transfected with control siRNA (siControl), LAMP3-specific siRNA (siLAMP3) and/or SPP1-specific siRNA (siSPP1) and the mRNA expression levels of MMP2, TWIST1, CDH1, CDH2, KRT19 and COL3A1 were examined by quantitative polymerase chain reaction. "P $<0.05$ and ${ }^{* *} \mathrm{P}<0.01$ vs. siControl. SPP1, secreted phosphoprotein 1; LAMP3, lysosomal-associated membrane protein 3; si, small interfering; MMP, matrix metallopeptidase; TWIST1, twist family bHLH transcription factor 1; CDH, cadherin; KRT19, keratin 19; COL3A1, collagen type III $\alpha 1$.

A

SPP1 gene ontology in osteosarcoma patients

GO:0001649: Osteoblast differentiation

GO:0007155: Cell adhesion

GO:0006954: Inflammatory response

GO:0007566: Embryo implantation

GO:0010811: Positive regulation of cell-substrate adhesion

GO:0030198: Extracellular matrix organization

G0:0030593: Neutrophil chemotaxis

G0:0031214: Biomineral tissue development

GO:0033280: Response to vitamin D

G0:0045780: Positive regulation of bone resorption

GO:0046697: Decidualization

GO:0048545: Response to steroid hormone stimulus

GO:0048685: Negative regulation of collateral sprouting of intact axon in response to injury

GO:0005576: Extracellular region

GO:0005615: Extracellular space

GO:0031988: Membrane-bounded vesicle

GO:0042995: Cell projection

GO:0045177: Apical part of cell

GO:0048471: Perinuclear region of cytoplasm

GO:0005125: Cytokine activity

GO:0050840: Extracellular matrix binding
B

The SPP1 regulated TOP KEGG

pathway in osteosarcoma patients

4510: Focal adhesion

4512: ECM-receptor interaction

4620: Toll-like receptor signaling pathway

Figure 5. Bioinformatics analysis of enriched cellular functions and pathways in osteosarcoma involving SPP1. (A) Gene ontology and (B) KEGG pathway analysis revealed that SPP1 may be involved in the osteoblast differentiation and cell adhesion functions and in the focal adhesion and ECM-receptor interaction pathways. SPP1, secreted phosphoprotein 1; KEGG, Kyoto Encyclopedia of Genes and Genomes; ECM, extracellular matrix.

chemotherapy, the survival rate of osteosarcoma is $<20 \%$ when patients are diagnosed with pulmonary metastasis or relapse (2). Thus, effective prevention of metastasis may help to improve the survival rate of patients with osteosarcoma. 
Metastasis is a complex process involving multiple factors and steps. Understanding the mechanisms underlying metastasis may help to develop novel therapeutic strategies for the improvement of patient outcome. Emerging evidence indicates that intratumoral hypoxia has important roles in cancer cell invasion and metastasis through the transcriptional activity of hypoxia-inducible factors, which affect the deposition, remodeling and degradation of ECM $(13,14)$. LAMP3, a member of the LAMP family, is induced by hypoxia and has been linked to metastasis of several types of cancers $(4,15)$. In the current study, LAMP3 was demonstrated to be significantly increased in osteosarcoma lung metastatic tumors, indicating a potential role of LAMP3 in osteosarcoma metastasis. Knockdown of LAMP3 reduced the invasiveness of osteosarcoma cells, further suggesting a premetastatic effect of LAMP3 in osteosarcoma cells. These results suggest that LAMP3 may be important in osteosarcoma metastasis.

Multiple different genes are involved in the regulation of cancer cell invasion. In the present study, knockdown of LAMP3 resulted in gene expression changes of a variety of genes, among which SPP1 was the most significantly increased gene detected. SPP1 is a well-studied protein that interacts with a number of integrin heterodimers to increase their activity and to regulate cell adhesion and motility, which are characteristic metastatic phenotypes of cancer cells. Several lines of evidence have demonstrated that upregulation of SPP1 induces metastasis indifferent types of cancer, including non-small cell lung cancer (16), prostate cancer (17), colon adenocarcinoma (18) and breast cancer (19). By contrast, the present results observed an elevated expression of SPP1 in the siLAMP3-attenuated invasive osteosarcoma cells, and knockdown of SPP1 rescued the reduced invasiveness of osteosarcoma cells and the gene expression alterations induced by LAMP3 knockdown. In addition, gene ontology and KEGG pathway analysis suggested that SPP1 was involved in osteoblast differentiation, cell adhesion, and ECM-receptor interaction, which are critical functions in cancer cell invasion. These results strongly suggest that SPP1 may act downstream of LAMP3 serving a protective role in osteosarcoma metastasis.

In addition to SPP1, changes in the expression of several genes were observed following LAMP3 knockdown. Cadherins belong to a class of cell adhesion molecules which regulate multiple aspects of cellular behavior, including cancer cell invasion. E-cadherin (encoded by the CHD1 gene) and $\mathrm{N}$-cadherin (encoded by the CHD2 gene) are two cadherins that have been widely investigated in cancer invasion and metastasis. $\mathrm{CDH} 1$ is a well-known cancer invasion suppressor gene in humans, while $\mathrm{CDH} 2$ promotes invasion in many types of cancer $(20,21)$. Downregulation of CDH1 is associated with increased invasion and metastasis in non-small cell lung cancer (22). In the present study, knockdown of LAMP3 resulted in the upregulation of CDH1 mRNA expression and E-cadherin protein expression, but downregulation of $\mathrm{CDH} 2 \mathrm{mRNA}$ expression, which is consistent with a reduced invasive ability of osteosarcoma cells. KRT19 is a biliary/hepatic progenitor cell marker. It has been reported that KRT19 is correlated with the invasion of human hepatocellular carcinomas (23). The present results detected increased KRT19 expression in osteosarcoma cells following LAMP3 knockdown, suggesting that KRT19 may also contribute to the invasion of osteosarcoma cells.

TWIST is a key transcription factor involved in epithelial-mesenchymal transition, a cellular dedifferentiation program that promotes invasion and metastasis (24). Knockdown of LAMP3 reduces the expression of TWIST1. Matrix metalloproteinases (MMPs) are matrix-degrading enzymes. MMP2 and MMP9 cleave type IV collagen, the major constituent of basement membrane, and serve critical roles in regulating the metastasis of osteosarcoma $(25,26)$. Thus, expression of MMP2 and MMP9 was also examined in LAMP3-silenced osteosarcoma cells. The results demonstrated that MMP2, but not MMP9, was downregulated in osteosarcoma cells following LAMP3 knockdown, suggesting that MMP2 may contribute to LAMP3-mediated invasion. COL3A1 is another important gene, which encodes the collagen chain of basement membrane, the critical component of ECM. Recently, Qiu et al (8) reported that COL3A1 is important in miR-29a/b-mediated migration and invasion of nasopharyngeal carcinoma. The present data demonstrated that LAMP3 knockdown reduced the expression of COL3A1, suggesting that COL3A1 may also contribute to LAMP3-mediated invasion in osteosarcoma cells.

In summary, LAMP3 mRNA expression is increased in lung metastatic tissues compared to primary tumor tissues of osteosarcoma. The present study demonstrated that knockdown of LAMP3 reduced the invasive ability of osteosarcoma cells via regulating multiple downstream genes involved in cell-cell adhesion and ECM homeostasis. The LAMP3/SPP1 signaling pathway might, therefore, function as a novel target for the prevention of invasion and metastasis of osteosarcoma.

\section{References}

1. Duong LM and Richardson LC: Descriptive epidemiology of malignant primary osteosarcoma using population-based registries, United States, 1999-2008. J Registry Manag 40: 59-64, 2013.

2. Daw NC, Chou AJ, Jaffe N, Rao BN, Billups CA, Rodriguez-Galindo C, Meyers PA and Huh WW: Recurrent osteosarcoma with a single pulmonary metastasis: A multi-institutional review. Br J Cancer 112: 278-282, 2015.

3. Ozaki K, Nagata M, Suzuki M, Fujiwara T, Ueda K, Miyoshi Y, Takahashi E and Nakamura Y: Isolation and characterization of a novel human lung-specific gene homologous to lysosomal membrane glycoproteins 1 and 2: Significantly increased expression in cancers of various tissues. Cancer Res 58: 3499-3503, 1998.

4. Kanao H, Enomoto T, Kimura T, Fujita M, Nakashima R, Ueda Y, Ueno Y,Miyatake T, Yoshizaki T, Buzard GS, et al: Overexpression of LAMP3/TSC403/DC-LAMP promotes metastasis in uterine cervical cancer. Cancer Res 65: 864ss0-8645, 2005.

5. Sun R, Wang X, Zhu H, Mei H, Wang W, Zhang S and Huang J: Prognostic value of LAMP3 and TP53 overexpression in benign and malignant gastrointestinal tissues. Oncotarget 5: 12398-12409, 2014.

6. Nagelkerke A, Mujcic H, Bussink J, Wouters BG, van Laarhoven HW, Sweep FC and Span PN: Hypoxic regulation and prognostic value of LAMP3 expression in breast cancer. Cancer 117: 3670-3681, 2011.

7. Liao X, Chen Y, Liu D, Li F, Li X and Jia W: High expression of LAMP3 is a novel biomarker of poor prognosis in patients with esophageal squamous cell carcinoma. Int J Mol Sci 16: 17655-17667, 2015.

8. Qiu F, Sun R, Deng N, Guo T, Cao Y, Yu Y, Wang X, Zou B, Zhang S, Jing T, et al: $\mathrm{miR}-29 \mathrm{a} / \mathrm{b}$ enhances cell migration and invasion in nasopharyngeal carcinoma progression by regulating SPARC and COL3A1 gene expression. PLoS One 10: e0120969, 2015. 
9. Nagelkerke A, Sieuwerts AM, Bussink J, Sweep FC, Look MP, Foekens JA, Martens JW and Span PN: LAMP3 is involved in tamoxifen resistance in breast cancer cells through the modulation of autophagy. Endocr Relat Cancer 21: 101-112, 2014.

10. Livak KJ and Schmittgen TD: Analysis of relative gene expression data using real-time quantitative PCR and the 2(-Delta Delta C(T)) method. Methods 25: 402-408, 2001.

11. Yu G, Wang LG, Han Y and He QY: clusterProfiler: An R package for comparing biological themes among gene clusters. OMICS 16: 284-287, 2012.

12. Liu K, He Q, Liao G and Han J: Identification of critical genes and gene interaction networks that mediate osteosarcoma metastasis to the lungs. Exp Ther Med 10: 1796-1806, 2015.

13. Finger EC and Giaccia AJ: Hypoxia, inflammation, and the tumor microenvironment in metastatic disease. Cancer Metastasis Rev 29: 285-293, 2010.

14. Gilkes DM, Semenza GL and Wirtz D: Hypoxia and the extracellular matrix: Drivers of tumour metastasis. Nat Rev Cancer 14 430-439, 2014

15. Mujcic H, Nagelkerke A, Rouschop KM, Chung S, Chaudary N, Span PN, Clarke B, Milosevic M, Sykes J, Hill RP, et al: Hypoxic activation of the PERK/eIF $2 \alpha$ arm of the unfolded protein response promotes metastasis through induction of LAMP3. Clin Cancer Res 19: 6126-6137, 2013.

16. Shojaei F, Scott N, Kang X, Lappin PB, Fitzgerald AA Karlicek S, Simmons BH, Wu A, Lee JH, Bergqvist $\mathrm{S}$ and Kraynov E: Osteopontin induces growth of metastatic tumors in a preclinical model of non-small lung cancer. J Exp Clin Cancer Res 31: 26, 2012

17. Ding Z, Wu CJ, Chu GC, Xiao Y, Ho D, Zhang J, Perry SR, Labrot ES, Wu X, Lis R, et al: SMAD4-dependent barrier constrains prostate cancer growth and metastatic progression. Nature 470: 269-273, 2011

18. Wai PY, Mi Z, Guo H, Sarraf-Yazdi S, Gao C, Wei J, Marroquin CE, Clary B and Kuo PC: Osteopontin silencing by small interfering RNA suppresses in vitro and in vivo CT26 murine colon adenocarcinoma metastasis. Carcinogenesis 26 741-751, 2005.
19. Mi Z, Guo H, Russell MB, Liu Y, Sullenger BA and Kuo PC: RNA aptamer blockade of osteopontin inhibits growth and metastasis of MDA-MB231 breast cancer cells. Mol Ther 17: 153-161, 2009.

20. Berx G, Staes K, van Hengel J, Molemans F, Bussemakers MJ, van Bokhoven A and van Roy F: Cloning and characterization of the human invasion suppressor gene E-cadherin (CDH1). Genomics 26: 281-289, 1995 .

21. Mareel M and Leroy A: Clinical, cellular, and molecular aspects of cancer invasion. Physiol Rev 83: 337-376, 2003.

22. Wu C, Zhu W, Qian J, He S, Wu C, Chen Y and Shu Y: WT1 promotes invasion of NSCLC via suppression of CDH1. J Thorac Oncol 8: 1163-1169, 2013.

23. Govaere O, Komuta M, Berkers J, Spee B, Janssen C, de Luca F, Katoonizadeh A, Wouters J, van Kempen LC, Durnez A, et al: Keratin 19: A key role player in the invasion of human hepatocellular carcinomas. Gut 63: 674-685, 2014.

24. Wang Y, Liu J, Ying X, Lin PC and Zhou BP: Twist-mediated epithelial-mesenchymal transition promotes breast tumor cell invasion via inhibition of hippo pathway. Sci Rep 6: 24606 , 2016.

25. Cho HJ, Lee TS, Park JB, Park KK, Choe JY, Sin DI, Park YY, Moon YS, Lee KG, Yeo JH, et al: Disulfiram suppresses invasive ability of osteosarcoma cells via the inhibition of MMP-2 and MMP-9 expression. J Biochem Mol Biol 40: 1069-1076, 2007.

26. Bjornland K, Flatmark K, Pettersen S, Aaasen AO, Fodstad O and Maelandsmo GM: Matrix metalloproteinases participate in osteosarcoma invasion. J Surg Res 127: 151-156, 2005. 